\title{
AN EXPLORATION OF THE RELATIONSHIP BETWEEN TIMING AND DURATION OF MAINTENANCE ACTIVITIES
}

RAM M. PENDYALA ${ }^{a} \&$ CHANDRA R. BHAT ${ }^{b}$

${ }^{a}$ Department of Civil and Environmental Engineering, University of South Florida, ENB 118, 4202 East Fowler Avenue, Tampa, FL 33620-5350, USA. Ph: (813) 974-1084; Fax: (813) 974-

2957; Email: pendyala@eng.usf.edu (corresponding author)

${ }^{b}$ Department of Civil Engineering, Ernest Cockrell Jr. Hall, 6.810, The University of Texas at Austin, Austin, Texas 78712, USA. Ph: (512) 471-4535; Fax: (512) 475-8744; Email:

bhat@mail.utexas.edu 


\section{ABSTRACT}

The timing and duration of an activity episode are two important temporal aspects of activitytravel behavior. Understanding the causal relationship between these two variables would be useful in the development of activity-based travel demand modeling systems. This paper investigates the relationship between these two variables by considering two different causal structures - one structure in which time-of-day choice is determined first and influences duration and a second structure in which activity duration is determined first and affects time-of-day choice. These two structures are estimated within a discrete-continuous simultaneous equations framework employing a full-information maximum likelihood methodology that allows error covariance. The estimation is performed separately for commuter and non-commuter samples drawn from a 1996 household travel survey data set from the Tampa Bay area in Florida. The results of the model estimation effort show that the causal structure in which activity duration precedes or affects activity timing (time of day choice) performs better for the non-commuter sample. For the commuter sample, the findings were less conclusive with both causal structures offering equally good statistical measures of fit. In addition, for the commuter sample, all error correlations were found to be zero. These two findings suggest that time of day choice and activity episode duration are only loosely related for the commuter sample, possibly due to the relatively non-discretionary and inflexible work activity and travel.

Keywords: activity duration, activity timing, activity-travel behavior, causal structure, simultaneous equations, time of day 


\section{INTRODUCTION}

Activity-based approaches to travel demand analysis explicitly recognize the important role played by time in shaping activity and travel patterns (Axhausen and Garling 1992). One of the key advantages of the activity-based approach is that it is capable of explicitly incorporating the time dimension into the travel modeling process (Pas and Harvey 1997). In the new planning context where travel demand management (TDM) strategies and transportation control measures (TCM) are inherently linked to the time dimension, activity-based approaches that recognize the time dimension offer a stronger behavioral framework for conducting policy analyses and impact studies (Bhat and Koppelman 1999; Harvey and Taylor 2000; Kitamura et al 1996; Pendyala et al 1997, 1998; Yamamoto and Kitamura 1999).

As an example of the importance of recognizing the time dimension, one may consider the case of telecommuting. When a worker telecommutes (from home), the commute to and from the work location is eliminated. Therefore, the worker now has additional time available for pursuing activities. The elimination of the commute trip influences the duration of travel and/or activity engagement. Besides influencing duration, telecommuting may influence the timing of activity engagement. Whereas a worker may have pursued non-work activities in combination with the commute when traveling to and from work, the worker may now choose to engage in non-work activities at other times of the day. In the absence of the commute trip, the worker no longer has the need or opportunity to link non-work activities to the commute. Analyzing these temporal changes in activity engagement patterns is important for accurately assessing the impacts of telecommuting on travel demand.

As illustrated by the telecommuting example, there are two key aspects of the temporal dimension that play an important role in activity-travel demand modeling (Goulias 1997). They 
are the timing of an activity episode and the duration (time allocation) of an activity episode (Mahmassani and Chang 1985; Mahmassani and Stephan 1988; Abkowitz 1981). In other words, activity-based analysis allows one to answer the two critical questions:

- When is an activity pursued?

- For how long is the activity pursued?

Recent activity-based research has focused on the analysis of individual activity episodes so that both of these aspects may be studied in detail (Bhat 1996, 1998; Bhat and Misra 1999; Bhat and Singh 2000). Studies that focused on daily time allocations to various activity types were not able to address the time-of-day choice in activity engagement (Kasturirangan et al 2002). Thus, conducting activity-based analysis at the individual activity episode level is crucial to gaining an understanding of the relationships between activity timing and duration (Hamed and Mannering 1993; Hunt and Patterson 1996; Levinson and Kumar 1995; Steed and Bhat 2000).

The relationship between activity timing and duration is an important component of activity-based travel demand modeling systems that aim to explicitly capture the temporal dimension (Kitamura et al 2000; Mannering et al 1994; Pendyala et al 2002; Wang 1996; Wen and Koppelman 2000). On the one hand, one may hypothesize that the timing of an activity affects its duration. Perhaps activity episodes pursued during peak periods are of short duration while those pursued in off-peak periods are longer in duration. On the other hand, the duration of an activity may affect its timing. Perhaps activities of longer duration are scheduled during the off-peak periods while activities of shorter duration are scheduled during peak periods. This paper attempts to shed light on this relationship by exploring both causal structures in a simultaneous equations framework. By identifying the causal structure that is most appropriate 
in different circumstances, one may be able to design activity based model systems that accurately capture the relationship between activity timing and duration.

This paper offers a detailed analysis of the relationship between activity timing and duration for maintenance activity episodes. The analysis is performed on commuter and noncommuter samples drawn from the 1996 Tampa Bay Household Travel Survey. A simultaneous equation system approach where activity timing is represented as a discrete time-of-day choice variable and duration is represented as a continuous variable is developed and estimated for two different causal structures. One causal structure assumes timing as a function of duration while the second assumes duration as a function of timing. The discrete-continuous simultaneous equations model offers a powerful framework for analyzing such causal structures (Hanemann 1984; Mannering and Hensher 1987; Barnard and Hensher 1992; Comte 1998).

Following a brief discussion in the next section, a description of the sample and data set is provided in the third section. The fourth section provides a descriptive analysis of activity timing and duration characteristics for maintenance activity episodes in the data set. The fifth section describes the modeling methodology and estimation procedure. The sixth and seventh sections provide results of the estimation of the two causal structures. Finally, the eighth section offers concluding remarks.

\section{THE ROLE OF TIME IN ACTIVITY-TRAVEL BEHAVIOR: TWO CAUSAL STRUCTURES}

Time is a finite and critical resource that is consumed in the engagement of activity and travel. All activities and trips consume time and regardless of the time span under consideration, there is only limited time within which an individual must pursue activities and trips. As travel 
is a derived demand, the focus of travel behavior research has shifted to analyzing the activity engagement patterns that drive trip making. This paper focuses on two critical temporal aspects of activity engagement, namely, the timing and duration of activities. The spatial dimension is very closely related to the temporal dimension as the distance traversed and the set of possible destination opportunities is dictated by timing and time availability. However, within the scope of this paper, only the temporal dimension and the causal relationships underlying activity timing and duration are explored.

To illustrate the importance of accurately capturing the relationship between activity timing and duration, two different causal structures may be considered in the context of analyzing the potential impacts of a variable pricing (congestion pricing or time-of-day based pricing) scheme. Such schemes are aimed at changing the time of travel or activity engagement so that trips otherwise undertaken during the congested peak periods would shift to off-peak periods. The two causal structures worthy of examination are briefly described in the following paragraphs.

\section{Causal Structure $D \rightarrow T$}

In this structure, activity episode duration is assumed to be predetermined. The timing of an activity is determined next and is dependent on the duration of the activity episode. The model system representative of this mechanism may be represented as follows:

$$
\begin{aligned}
& D_{a}{ }^{*}=\beta_{a}{ }^{\prime} X+\alpha_{a}{ }^{\prime} Z_{a}+\varepsilon_{a} \\
& T_{a}{ }^{*}=\delta_{a}{ }^{\prime} S+\omega_{a}{ }^{\prime} R_{a}+\theta_{a} D_{a}+v_{a} P+\xi_{a}
\end{aligned}
$$

where $D_{a}{ }^{*}=$ latent variable underlying episode duration for activity type a

$T_{a}{ }^{*}=$ latent variable underlying activity timing for activity type a

$X, S=$ vectors of socio-economic characteristics 
$Z_{a}, R_{a}=$ vectors of characteristics of activity type $a$

$D_{a}=$ observed or measured counterpart of $D_{a}{ }^{*}$

$P=$ variable pricing (amount charged)

$\varepsilon_{a}, \xi_{a}=$ random error terms that may be correlated

$\beta_{a}, \alpha_{a}, \delta_{a}, \omega_{a}, \theta_{a}, v_{a}=$ model coefficients

Thus, in this model structure, activity episode duration is modeled as a function of socioeconomic characteristics (that do change based on the activity type) and activity characteristics (different activity types may have different characteristics). The time of day choice is then modeled as a function of socio-economic characteristics, activity characteristics, the variable pricing cost, and the duration of the activity episode. Thus, in this scheme, the duration of the activity is predetermined and the timing is determined as a function of the duration. As variable pricing is aimed at merely shifting time of travel, it appears as an explanatory variable only in the timing equation.

\section{Causal Structure $T \rightarrow D$}

In the second causal structure, the time of day choice for an activity episode is determined first. The duration of an activity episode is determined second in the causal structure. The simultaneous equation system representative of this causal scheme is as follows:

$$
\begin{aligned}
& T_{a}{ }^{*}=\delta_{a}{ }^{\prime} \mathrm{S}+\omega_{a}{ }^{\prime} R_{a}+\nu_{a} P+\xi_{a} \\
& D_{a}{ }^{*}=\beta_{a}{ }^{\prime} X+\alpha_{a}{ }^{\prime} Z_{a}+\theta_{a} T_{a}+\varepsilon_{a}
\end{aligned}
$$

All of the symbols are as described previously. In this scheme, activity timing is a function of socio-economic characteristics, activity characteristics, and variable pricing. After the timing has been determined, the activity episode duration is determined as a function of socio-economic 
characteristics, activity characteristics, and activity timing. Once again, variable pricing appears only in the timing equation.

Now, suppose one is interested in determining the potential impacts of variable pricing on travel demand by time of day. The implications of using the two different structures for impact assessment are very significant. In causal structure $D \rightarrow T$, duration is predetermined and is not sensitive to time of day choice. In the presence of variable pricing, the extent to which a shift in activity timing may take place is dependent on the activity duration. In causal structure $T \rightarrow D$, timing is sensitive to variable pricing and is determined first. The activity episode duration is then adjustable in response to the timing of the activity episode. Thus, the duration is no longer fixed and does not affect the potential shift in timing.

In other words, if one used causal structure $D \rightarrow T$ to assess variable pricing impacts when in fact structure $T \rightarrow D$ is the correct one, then one might underestimate the potential shift in traffic. This is because timing is a function of duration and the duration itself is not responsive to variable pricing. So, even though the variable pricing cost may motivate an individual to shift time of travel for an activity, the duration of the activity may preclude the person from doing so. Thus causal structure $D \rightarrow T$ may inhibit the potential shift in timing. On a similar note, if one used causal structure $T \rightarrow D$ to assess variable pricing impacts when in fact structure $D \rightarrow T$ is the correct one, then one might overestimate the potential shift in traffic.

The above example shows the critical importance of identifying the appropriate causal structure that should be employed under different circumstances. It is possible that different causal structures would be suitable to different market segments, activity types, and urban contexts. This paper attempts to control for some of these aspects by considering activity timing and duration relationships only for maintenance activities. Models are estimated for commuters 
and non-commuters separately to control for the significant influence that work and commute episodes may have on activity timing and duration decisions.

\section{DATA SET AND SAMPLE COMPOSITION}

The data set is derived from a comprehensive household travel survey that was administered in 1996 in the Tampa Bay Region of Florida. The survey was a traditional trip diary survey and was not an activity or time use survey. The survey was a mail-out mail-back survey that collected household and person socio-economic and demographic characteristics together with detailed information about all trips undertaken over a 24 hour period. Households were asked to return one complete diary for every household member (including children); however, as expected, many households returned fewer diaries than household members. The survey instrument was mailed to about 15,000 households and over 5,000 households returned at least one trip diary resulting in a response rate close to 35 percent. Given the mail-out mail-back nature of the survey, this response rate may be considered quite reasonable and consistent with expectations.

After extensive checking and data integrity screening, a final respondent sample of 5261 households was obtained. From these 5261 households, a total of 9066 persons returned usable trip diaries. The 9066 persons reported information for a total of 31459 trips (through the 24 hour trip diary). The trip file was used to create an out-of-home activity file where individual activity records were created from the trip records. This activity file included information about activity type, activity timing, activity duration, and other variables pertinent to each activity episode. As the focus of this paper is on the modeling of causal relationships, the unweighted 
sample was considered sufficient for analysis purposes. No weighting or expansion of the sample has been done to reflect population characteristics.

This paper focuses on the relationship between activity timing and duration for maintenance activities. Maintenance activities included the following three activity (trip) types:

- Shopping, personal business, and errands

- Medical/dental

- Serve passenger or child

These activity records were extracted from the original file to create two maintenance activity record files, one for commuters and one for non-commuters. Commuters were defined as driving age individuals who commuted to a work place on the travel diary day, while noncommuters were defined as driving age individuals who did not commute to a work place (made zero work trips) on the travel diary day. Note that a worker (employed person) who did not commute on the travel diary day would still be classified as a non-commuter for the purpose of this paper. Also, children under the age of 16 were excluded from the analysis completely. Maintenance activity records that had full information (no missing data) were extracted to create commuter and non-commuter data files for the modeling effort in this paper.

Maintenance activities were pursued by 2904 individuals residing in 2386 households. Of these individuals, 1023 were commuters and they reported 1351 maintenance activities. The remaining 1881 individuals were non-commuters and they reported 2899 maintenance activities. The commuter and non-commuter maintenance activity episode data sets included complete socio-economic and activity information for the respective samples. For these specific data sets, Table 1 provides a summary of the sample composition together with average activity characteristics. This sample represents a self-selected sample of individuals who actually 
participated in a maintenance activity on the travel survey day. Thus, in modeling the relationship between activity timing and duration for these data sets, one needs to account for self-selectivity arising from the activity record selection and extraction process.

\section{[INSERT TABLE 1 ABOUT HERE]}

The average household size for the sample of 2386 households is 2.3 persons per household. More than one-half of the households are two-person households in this particular sample. Average vehicle ownership is about 1.8 vehicles per household with a little more than 40 percent of the sample owning two cars. More than three-quarters of the sample resides in a single family dwelling unit. About one-third of the sample has annual income less than $\$ 25,000$ while about one-quarter of the sample has an annual income greater than $\$ 50,000$.

The major differences between commuters and non-commuters seen in the age and employment distribution are consistent with expectations. Commuters are predominantly in the age groups of 22-49 years and 50-64 years while non-commuters are older with more than 60 percent greater than or equal to 65 years of age. Similarly, 80 percent of commuters are employed full time while only 7.7 percent of non-commuters are employed full time.

Among those who undertake at least one maintenance activity, non-commuters undertake (on average) a higher number of maintenance activities. In addition, they allocate more time to maintenance activities and have longer maintenance activity episode durations than commuters. While the commuter sample which reported at least one maintenance activity spent (on average) 1.5 hours for maintenance activities during the day, the non-commuter sample spent nearly 4 hours. On average, the commuter sample reported activity episode durations of 70 minutes while the non-commuter sample reported activity episode durations twice that amount at a little over 140 minutes. 


\section{DESCRIPTIVE ANALYSIS OF ACTIVITY DURATION AND TIMING}

Prior to commencing the model development effort, further analysis of the potential relationship between activity duration and timing was undertaken. The analysis presented in this section constitutes a cross-tabulation where the distributions of activity duration and activity timing are examined relative to one another. The results are presented in graphical form in Figures 1-4.

Based on a time of day distribution of all trips in the data set, four distinct time periods were identified. They are:

- AM peak: 7:15 AM - 9:15 AM

- Midday: 9:16 AM-3:15 PM

- PM peak: 3:16 PM - 6:15 PM

- Off peak: 6:16 PM - 7:14 AM

[INSERT FIGURES 1-4 ABOUT HERE]

Similarly, for tabulation and graphical representation purposes, the activity episode duration was categorized into five possible duration categories. Figure 1 shows the distribution of activity episode durations by time of day for commuters while Figure 2 shows a similar distribution for non-commuters. A comparison between these figures shows differences between commuters and non-commuters that are consistent with expectations. First, it is found that commuters undertake only about 30 percent of their maintenance activities in the midday (395 of 1351) while non-commuters undertake about 70 percent of their maintenance activities in the midday (2019 of 2899). In general, commuters show a greater proportion of maintenance activities in the PM peak period (presumably in conjunction with the return commute) and the 
off-peak period when compared with the non-commuters. The distribution of activity episode durations within each time period also reveals interesting differences. More than 60 percent of all AM peak maintenance activities are 1-10 minutes long for commuters; the corresponding percentage for non-commuters is only 20 percent. At the other end, about 13 percent of off-peak maintenance activities are more than 2 hours long for commuters; the corresponding percentage for non-commuters is 50 percent.

Figures 3 and 4 show the relationship between activity episode duration and activity timing in a way that presents the time of day distribution of activity episodes by activity duration. Once again, differences between commuters and non-commuters are striking and consistent with expectations. While 30 percent of maintenance activities are 1-10 minutes long for commuters (407 of 1351), the corresponding percentage for non-commuters is only 17 percent (493 of 2899). Similarly, while nearly one-quarter of maintenance activity episodes are more than two hours long for non-commuters, the corresponding percentage for commuters is only 11 percent. The differences in the time of day distributions are also quite striking. Whereas commuters tend to have a more even distribution among the midday, PM peak, and off peak periods, the non-commuters are extremely midday oriented in their maintenance activity engagement regardless of the duration of the activity.

Thus it appears that commuters tend to undertake shorter maintenance activities and evenly distribute them across the midday, PM peak, and off peak periods. For example, while commuters are pursuing about $30-40$ percent of their maintenance activities in the PM peak period, non-commuters are pursuing only about 10-20 percent of their maintenance activities in this period. Non-commuters are found to pursue maintenance activities of longer duration and tend to pursue such activities largely in the midday period. There is some tendency to shift the 
activity engagement to the off peak period for activities greater than 2 hours in duration. But, even for such long duration activities, more than 60 percent are pursued in the midday period. Activities pursued by commuters in the AM peak period tend to be of very short duration (1-10 minutes long). On the other hand, activities pursued by non-commuters in the off-peak period tend to be longer in duration.

The charts presented in this section show that commuters and non-commuters exhibit substantial differences in their activity timing and duration patterns. Therefore, separate models are developed in this paper for commuters and non-commuters. It is plausible that different causal structures may apply for these two different groups. Within each group, it is found that there are differences across time of day distributions by activity duration and activity duration distributions by time of day. Such differences point to the inter-relationship and activity timing and duration that is best modeled using a simultaneous equations framework. The next section provides a detailed description of the simultaneous equations methodology adopted in this paper.

\section{MODELING METHODOLOGY AND ESTIMATION PROCEDURE}

The decisions regarding the time of day of activity participation and activity duration are modeled using a joint discrete/continuous econometric framework. In such joint systems, logical consistency considerations require certain restrictions to be maintained on the coefficients representing the causal effects of the dependent variables on one another. Specifically, in the context of the joint time of day of activity participation and activity duration model of the current paper, the restrictions imply a recursive causal model in which time of day of activity participation affects activity duration or vice versa (but not both). The next subsection discusses the restrictions in more detail for the case of a simple binary choice for time of day, as it 
simplifies the presentation. However, the same restrictions extend to the case of a multinomial choice situation for time of day. Following this discussion, the structure and estimation technique for a multinomial time of day and continuous activity duration model are presented.

\section{Logical Consistency Condition}

Let $s^{*}$ be a latent continuous variable that determines an observed binary variable $s$ representing the time of day of activity participation; $s$ may take the value 0 (say AM participation) or 1 (say PM participation). Let $a$ be the logarithm of the duration of activity participation (the logarithm form guarantees the non-negativity of duration predictions).

Consider the following equation system, where the index for observations has been suppressed:

$$
\begin{aligned}
& s^{*}=\beta^{\prime} z+\delta_{1} a+\varepsilon, s=0 \text { if } s^{*} \leq 0 ; s=1 \text { if } s^{*}>0 \\
& a=\theta^{\prime} x+\delta_{2} s+\omega,
\end{aligned}
$$

where $z$ and $x$ are vectors of observed variables, $\varepsilon$ and $\omega$ are random error terms assumed to be normally distributed, and $\beta, \theta, \delta_{1}$, and $\delta_{2}$ are coefficients to be estimated. Using the second equation to replace a in the first equation, we obtain:

$$
s^{*}=\beta^{\prime} z+\delta_{1} \theta^{\prime} x+\delta_{1} \delta_{2} s+\delta_{1} \omega+\varepsilon, s=0 \text { if } s^{*} \leq 0 ; s=1 \text { if } s^{*}>0 .
$$

From the above equation, one can write the following:

$$
\begin{aligned}
& \operatorname{Prob}[s=0]=1-\Phi\left(\beta^{\prime} z+\delta_{1} \theta^{\prime} x\right) \\
& \operatorname{Prob}[s=1]=\Phi\left(\beta^{\prime} z+\delta_{1} \theta^{\prime} x+\delta_{1} \delta_{2}\right)
\end{aligned}
$$

where $\Phi$ is the cumulative normal distribution function of $\delta_{1} \omega+\varepsilon$. The sum of the above two probabilities is 1 only if $\delta_{1} \delta_{2}=0$; that is, only if either $\delta_{1}=0$ or $\delta_{2}=0$ in Equation (1). Intuitively, the logical consistency condition $\delta_{1} \delta_{2}=0$ states that $s^{*}$ cannot be determined by $s$ if it also determines $s$ (see Equation 2) (Maddala 1983). 
The application of the logical consistency condition leads to a recursive model system. If $\delta_{1}=0$, then the time of day of participation affects the logarithm of activity duration (but not vice versa). If $\delta_{2}=0$, then the logarithm of activity duration affects time of day of participation (but not vice versa). A natural question then is: which of the two assumptions $\left(\delta_{1}=0\right.$ or $\left.\delta_{2}=0\right)$ should be maintained? In this paper, both recursive systems are estimated and the two alternative systems are tested empirically to provide guidance regarding the causal direction to maintain in a joint time of activity participation and activity duration model system (Ben-Akiva and Lerman 1985; Van Garderen 2001).

\section{Model Structure and Estimation Procedure}

The previous section used a binary choice structure for time of day to discuss the need to maintain a recursive structure in the time of day/duration model system. The same arguments are applicable even for a multinomial choice context for time of day. In the next section, the recursive structure in which time of day affects activity duration is discussed. Following this discussion, the alternative structure in which activity duration affects time of day is discussed.

\section{Time of Day Affects Activity Duration}

Let $i$ be an index for time of day of activity participation $(i=1,2, \ldots, I)$ and let $q$ be an index for observations $(q=1,2, \ldots, \mathrm{Q})$. Consider the following equation system:

$u_{q i}^{*}=\beta_{i}^{\prime} z_{q i}+\varepsilon_{q i}, \varepsilon_{q i} \sim \operatorname{IID~Gumbel}(0,1)$

$a_{q}=\theta^{\prime} x_{q}+\delta D_{q}+\omega_{q}, \omega_{q} \sim N\left(0, \sigma^{2}\right)$

where $u_{q i} *$ is the indirect (latent) utility associated with the ith time of day for the $q$ th observation, $D_{q}$ is a vector of the time of day dummy variables of length $\mathrm{I}, \delta$ is a vector of coefficients representing the effects of different times of the day of activity participation on 
activity duration, $\varepsilon_{q i}$ is a standard extreme-value (Gumbel) distributed error term assumed to be independently and identically distributed across times of the day and observations, and other variables are as defined earlier in Equation (1) with the addition of appropriate subscripts. The error term $\omega_{q}$ is assumed to be identically and independently normal-distributed across observations with a mean of zero and variance of $\sigma^{2}$.

In Equation (4), the time of day alternative $i$ will be chosen (i.e., $D_{q i}=1$ ) if the utility of that alternative is the maximum of the I alternatives. Defining $v_{q i}=\left\{\begin{array}{c}\max u_{q j} * \\ j=1,2, \ldots, I, j \neq i\end{array}\right\}-\varepsilon_{q i}$,

the utility maximizing condition for the choice of the $i$ th alternative may be written as: $D_{q i}=1$ if and only if $\beta_{i}^{\prime} z_{q i}>v_{q i}$. Let $F_{i}\left(v_{q i}\right)$ represent the marginal distribution function of $v_{q i}$ implied by the assumed IID extreme value distribution for the error terms $\varepsilon_{q i}(i=1,2, \ldots, \mathrm{I})$ and the relationship in Equation (5). Using the properties that the maximum over identically distributed extreme value random terms is extreme value distributed and the difference of two identically distributed extreme values terms is logistically distributed, the implied distribution for $v_{q i}$ may be derived as:

$$
F_{i}(y)=\operatorname{Prob}\left(v_{q i}<y\right)=\frac{\exp (y)}{\exp (y)+\sum_{j \neq i} \exp \left(\beta_{j}^{\prime} z_{q j}\right)} .
$$

Following Lee (1983), the non-normal variable $v_{q i}$ is transformed into a standard normal variate using the integral transform result (Feller 1971):

$v_{q i} *=\Phi^{-1}\left[F_{i}\left(v_{q i}\right)\right]$, 
where $\Phi($.$) is the standard cumulative distribution function. Equation system (4) may now be$ rewritten as:

$$
\begin{aligned}
& D_{q i}^{*}=\beta_{i}^{\prime} z_{q i}-v_{q i}^{*}, D_{q}=0 \text { if } D_{q i}^{*}<0, D_{q}=1 \text { if } D_{q i}^{*}>0 \\
& a_{q}=\theta^{\prime} x_{q}+\delta^{\prime} D_{q}+\omega_{q}
\end{aligned}
$$

A correlation $\rho_{i}$ between the error terms $v_{q i} *$ and $\omega_{q}$ is allowed to accommodate common underlying unobserved factors influencing the time of day choice for activity participation and the duration of the participation. For example, individuals who are physically challenged or "take things slowly" may prefer to participate in activities during the midday periods (rather than early in the morning) and may also have a long duration of participation.

The parameters to be estimated in the joint model system are the $\beta_{i}$ parameter vectors in the time of day choice model, the $\theta$ and $\delta$ parameter vectors in the activity duration model, the standard deviation $\sigma$ of the $\omega_{q}$ random term, and the correlation parameters $\rho_{i}$. The likelihood function for estimating these parameters is:

$L=\prod_{q=1}^{Q}\left\{\prod_{i=1}^{I}\left[\frac{1}{\sigma} \phi\left(l_{q}\right) \Phi\left(b_{q i}\right)\right]^{D_{q i}}\right\}$

where $\phi($.$) is the standard normal density function, and l_{q}$ and $b_{q i}$ are defined as follows:

$$
l_{q}=\left(\frac{a_{q}-\theta^{\prime} x_{q}-\delta D_{q}}{\sigma}\right), b_{q i}=\left(\frac{\Phi^{-1} F_{i}\left(\beta_{i}^{\prime} z_{q i}\right)-\rho_{i} l_{q}}{\sqrt{1-\rho_{i}^{2}}}\right)
$$

\section{Activity Duration Affects Time of Day}

The equation system in this case may be written in the following form:

$$
\begin{array}{ll}
u_{q i}{ }^{*}=\beta_{i}^{\prime} z_{q i}+\gamma a_{q}+\varepsilon_{q i}, & \varepsilon_{q i} \sim \operatorname{IID~Gumbel}(0,1) \\
a_{q}=\theta^{\prime} x_{q}+\omega_{q}, & \omega_{q} \sim N\left(0, \sigma^{2}\right) .
\end{array}
$$

The above system can be rewritten using the same procedures as in the previous section as: 
$D_{q i}{ }^{*}=\beta_{i}^{\prime} z_{q i}+\gamma a_{q}-v_{q i}{ }^{*}, D_{q i}=0$ if $D_{q i}{ }^{*}<0, D_{q i}=1$ if $D_{q i}{ }^{*}>0$

$a_{q}=\theta^{\prime} x_{q}+\omega_{q}$.

Assuming a correlation $\rho_{i}$ between $v_{q i}{ }^{*}$ and $\omega_{q}$, the likelihood function for estimating the parameters $\beta_{i}(i=1,2, \ldots I), \gamma, \theta, \sigma$, and $\rho_{i}$ is exactly the same as in Equation (9) with the following alternative definitions for $l_{q}$ and $b_{q i}$ :

$l_{q}=\left(\frac{a_{q}-\theta^{\prime} x_{q}}{\sigma}\right), b_{q i}=\left(\frac{\Phi^{-1} F_{i}\left(\beta_{i}^{\prime} z_{q i}+\gamma a_{q}\right)-\rho_{i} l_{q}}{\sqrt{1-\rho_{i}^{2}}}\right)$.

\section{ESTIMATION RESULTS: NON-COMMUTERS}

This section provides results of the model estimation effort for the non-commuter sample. Table 2 shows estimation results for the joint model where time of day choice affects activity duration. Table 3 shows estimation results for the joint model where activity duration affects time of day choice. The first block in each table corresponds to the time of day choice model where the off-peak period is considered the base alternative. The second block corresponds to the log-linear duration model. The standard deviation, $\sigma$, of the error term in the log-linear duration model is also provided. Finally, correlations between error terms that turned out to be significant are documented.

[INSERT TABLES 2 AND 3 ABOUT HERE]

In Table 2, various socio-demographic variables are found to influence time of day choice. The alternative specific constants in the time of day model seem to suggest that, relative to the off-peak period, there is a greater likelihood of pursuing a maintenance activity episode in the AM-peak, midday, and PM-peak periods. All of the alternative specific constants are positive and significant. The greatest likelihood occurs in the midday period. While the 
propensity to pursue a maintenance activity in the midday period is quite plausible for the noncommuter sample, the positive alternative specific constants associated with the peak periods are less plausible. One would expect non-commuters to pursue maintenance activities in the midday and off-peak periods.

Non-commuters aged 50-64 years appear to show a greater propensity to pursue maintenance activities in the off-peak period. All of the coefficients appearing in the model are negative. After the off-peak period, the preferred time slots appear to be the midday and PMpeak periods. A full-time employed person who did not commute on the survey day tends to perform a maintenance activity in the AM-peak period. This may be a serve-child trip or another habitual trip that the worker performs regardless of whether he/she commutes on a particular day. A homemaker shows a greater propensity to pursue maintenance activities in the midday period. Single persons (household size $=1$ ) show a greater propensity to pursue maintenance activities in the PM-peak period, possibly because they combine such activities with other recreational activities.

In the duration model, it can be seen that maintenance activity episodes tend to be longer for single persons, persons living in households with no children, and persons living in lower income households. It is possible that these persons are less rushed and therefore are able to pursue their maintenance activity episodes in a more relaxed manner. On the other hand, a homemaker and a person aged 22-49 years have negative coefficients reflecting shorter maintenance activity episode lengths. These individuals may be those who have household and child obligations that contribute to shorter maintenance activity episodes. The time of day indicators are all statistically significant in the model. Relative to the off-peak period, all other periods are characterized by shorter maintenance activity episodes as reflected by the negative 
coefficients. Strangely, the midday indicator has the most negative coefficient suggesting that the maintenance activity episodes in this period are the shortest.

In this model, the error correlation between midday activity participation and activity duration is the only statistically significant error correlation. The coefficient suggests that there is a positive correlation between midday activity participation and activity duration during this period (note that the negative sign in equation 8 and the negative sign of the estimate combine to form the positive correlation). This positive correlation is quite intuitive. It indicates that unobserved factors that increase the likelihood of participation during the midday also increase activity duration during that period. For example, non-commuters who are physically challenged or who "take things slow" might not want to leave home early in the morning and might avoid the evening peak and late night due to congestion or safety considerations. Such individuals would prefer midday activity participation and the same unobserved factors of "physically challenged" or "wanting to take things slow" would lead to long durations of activity participation.

In Table 3, the alternative specific constants in the time of day choice model show that, relative to the off-peak period, there is a smaller likelihood of pursuing a maintenance activity episode in the AM-peak and PM-peak periods and a greater likelihood of pursuing a maintenance activity in the midday period. This finding is very logical for a non-commuter sample that is likely to pursue maintenance activities in the midday period and off-peak period. The activity duration variable affects time of day choice in the AM peak and PM peak activity participation equations. It does not enter the midday activity participation equation. The coefficients associated with the duration variable are positive indicating that non-commuters are not constrained with respect to the lengths of their activity episodes in these time periods. They can 
pursue long maintenance activity episodes in the peak periods just as in the midday or off-peak periods (Figure 2 also shows this). The dummy variables associated with age show negative coefficients indicating a greater propensity for most non-commuter adults to pursue maintenance activities in the off-peak period. Full-time employed individuals who did not commute on the survey day show a greater propensity to pursue maintenance activities in the AM-peak period, possibly due to a habit effect.

The log duration model also offers plausible indications. Single persons and persons in households with no child offer positive coefficients indicating the ability to engage in longer maintenance activity episodes relative to homemakers and persons aged 22-49 years. In both Tables 2 and 3, it can be seen that the history of maintenance activity engagement up to the current activity has a positive coefficient. This suggests that, for non-commuters, there is a complementary effect across maintenance activity episodes. Non-commuters, who might be typically bearing the household maintenance activity responsibilities, have a positive codependence between maintenance activity episodes. As in Table 2, the negative error correlation term suggest a positive correlation between midday activity participation and episode duration.

As the time of day indicator variables were significant in Table 2 and the duration variables were significant in Table 3, an assessment of the causal structures may be performed through the goodness-of-fit measures. Table 6 offers goodness-of-fit measures for the two noncommuter models. From these measures (and the interpretations of the alternative specific constants and coefficients), it is found that the model represented in Table 3, namely, activity duration affects time of day choice is performing better than that represented in Table 2 (in which time of day affects activity duration). For non-commuters, it appears that the causal 
decision structure is one in which the activity episode duration is estimated by an individual and then scheduled (timed) based on that best guess estimate of the duration.

The adjusted likelihood ratio index at zero is computed as:

$$
\bar{\rho}_{0}^{2}=1-\frac{L(\beta)-k}{L(0)}
$$

where $\mathrm{k}$ is the number of parameters as shown in the table. Similarly, the likelihood ratio index at sample shares is computed as:

$$
\bar{\rho}_{c}^{2}=1-\frac{L(\beta)-k}{L(C)}
$$

A comparison of the adjusted likelihood ratio indices provides a mechanism for comparing two non-nested models. Ben-Akiva and Lerman [28] note that, for estimations involving more than 250 observations, if the adjusted likelihood ratio indices differ by more than 0.01, then the model with the lower index is almost certainly the incorrect model. As the difference in indices for the non-commuter models is substantially greater than 0.01 , it may be safely concluded that the model representing the causal structure where duration affects time of day is the more appropriate one.

\section{ESTIMATION RESULTS: COMMUTERS}

This section provides results of the model estimation effort for the commuter sample. Table 4 shows estimation results for the joint model where time of day choice affects activity duration. Table 5 shows estimation results for the joint model where activity duration affects time of day choice. The structure of the tables is similar to that in Tables 2 and 3.

[INSERT TABLES 4 AND 5 ABOUT HERE] 
A key finding of the model estimation for commuters is that none of the error correlation terms are statistically significant regardless of the causal structure considered. Thus, in effect, the joint model reduces to an independent model system where time of day and duration models may be estimated separately in a sequential fashion. This is a key finding suggesting that there is only a loose relationship between time of day choice and activity episode duration for commuters.

In Table 4, the causal structure where time of day affects activity duration is considered. The alternative specific constants suggest that commuters tend to pursue the maintenance activities in the midday or PM-peak periods (possibly linked to the work activity/trip) as opposed to the AM- peak or off-peak periods. As expected, part-time employed persons show a greater propensity to pursue maintenance activities in the midday or PM peak periods. Homemakers who commute to a work place (possibly for volunteer activities) indicate a greater propensity to pursue maintenance activities in the midday. In general, commuting adults (aged 22-64 years) show a lower propensity to pursue maintenance activities during the work hours, possibly because of constraints imposed by work schedules.

The log-linear duration model in Table 4 shows that time of day variables are significant in influencing activity duration. As expected, episode duration tends to be longer in the off-peak period as reflected by the positive coefficient associated with the off-peak period indicator. In general, persons who are full-time employed, 22-49 years old, and in multi-person households show a propensity to engage in shorter activity episodes. Those who live alone (household size =1) exhibit a positive coefficient indicating they are less rushed and engage in longer activity episodes. 
In Table 5, the causal structure where activity duration affects time of day choice is considered. Activity duration is found to be significant in all time period equations. Relative to the off-peak period, the negative coefficients associated with activity duration suggest that there is lower propensity to pursue longer activity episodes in the AM-peak, midday, or PM-peak periods. Within these three periods, the lowest propensity is seen in the AM-peak period and the highest propensity is seen in the midday periods. These findings are consistent with expectations. Full-time workers are generally more likely to pursue maintenance activities in the off-peak period as reflected by the negative coefficients associated with the worker and full-time employed dummy variables. On the other hand, a part-time employed person shows a greater propensity to perform maintenance activities in the midday or AM-peak periods. Part-time workers may have the flexibility that allows such activity engagement patterns.

The log-linear duration model in Table 5 shows that full-time employed persons and those living in multi-person households or in middle income households have shorter maintenance activity episodes. Those living alone (household size $=1$ ) have longer maintenance activity episodes, possibly due to the lack of other household member obligations and constraints.

\section{[INSERT TABLE 6 ABOUT HERE]}

In general, both Tables 4 and 5 show slightly weaker interpretations relative to the noncommuter models. Within each causal structure, the time of day variables or the duration variable are significant in the respective equation suggesting that there is a strong correlation between these decisions. However, a review of the goodness-of-fit measures presented in Table 6 for the commuter models shows that it is very difficult to identify a better or more appropriate causal structure for commuters. Both models offer virtually identical goodness-of-fit measures. 
An examination of Table 6 shows that the adjusted likelihood ratio indices are very similar and have differences less than the 0.01 value required to help identify the correct model. In addition, the model fit is substantially poorer than the fits obtained in the context of the non-commuter samples. These findings coupled with the finding that none of the error correlation terms are statistically significant suggest that time of day choice and activity episode duration are correlated albeit with only a loose causal relationship between them. It does not appear that one decision precedes or necessarily determines the other. This conclusion may be explained by the fact that work schedules tend to dictate time of day participation and activity durations for commuters. Given work schedules and constraints, there is not much flexibility for commuters to exercise choices and go through a decision process characterized by choices and causal relationships.

\section{DISCUSSION AND CONCLUSIONS}

This paper has presented an exploration of the relationship between activity timing (time of day choice) and activity episode duration for maintenance activities such as shopping, personal business, errands, and others. The analysis involved the estimation of joint models of activity timing and duration separately for commuters and non-commuters while allowing error correlations between the timing and duration model equations. Time of day choice was modeled as a discrete choice variable involving four alternative periods of the day while duration was modeled using a log-linear formulation.

Travel survey data from the 1996 household travel survey conducted in the Tampa Bay area of Florida was used to describe the relationships between activity timing and duration and estimate the models. Two different causal structures were considered: 
- Activity timing (time of day choice) affects activity duration

- Activity episode duration affects activity timing (time of day choice)

Both of these causal structures were estimated on the non-commuter and commuter sample activity episodes to identify the appropriate causal structure for each sample group. The identification of such causal relationships between activity engagement phenomena is very important from several key perspectives. First, the identification of appropriate causal structures will help in the development of accurate activity-based travel demand model systems that intend to capture such relationships at the level of the individual traveler and activity episode. Second, a knowledge of the true causal relationships underlying decision processes will help in the accurate assessment and impact analysis of alternative transportation policies such as variable pricing, parking pricing, and telecommuting.

For the non-commuter sample, it was found that the model in which activity duration is assumed to be determined first and then influence time of day choice offered superior statistical measures of fit than the model in which activity timing was assumed to precede and determine activity duration. In addition, the non-commuter model showed a significant error correlation between midday activity participation and activity episode duration suggesting non-commuters who do not like to be rushed prefer to engage in longer activities during the midday (avoiding peak periods). These findings suggested that activity timing and duration are closely related for the non-commuter sample and that activity duration precedes the choice of time of day.

For the commuter sample, on the other hand, it was found that both causal structures offered virtually identical statistical measures of fit and that the fits were substantially poorer than those obtained for the non-commuter samples. In addition, all of the error correlation terms were found to be statistically insignificant suggesting that activity timing and duration could be 
modeled in an independent and sequential framework. These findings suggest that activity timing and duration are only loosely related from a causal decision-making standpoint although they are correlated with one another. Commuters, who have relatively more constraints imposed by work schedules and commute trips, may not have the ability to exercise a decision process that is characterized by choices, alternatives, and causal relationships. In such a context, it is very difficult to identify a causal structure or relationship underlying activity timing and duration.

The use of a traditional trip diary-based survey data set for the analysis proposed in this paper does not come without limitations. One of the main limitations is that a trip diary survey does not capture multiple activity episodes that may occur at the same location (either simultaneously or sequentially). A trip diary survey captures only one activity per location or trip. Thus, it is possible that the trip diary survey data used in the analysis of this paper does not capture all of the individual activity episodes of maintenance activities. More comprehensive activity or time use-based surveys should be used in the future to investigate the robustness of the findings reported in this paper.

Activity scheduling surveys that involve the collection of data on underlying behavioral processes make it possible to study timing decisions in a robust framework (Doherty and Miller 2000). Such data would greatly help further explore the causal linkages between timing and other activity-travel variables. In addition, such data would help further explore the decision processes that govern activity-travel engagement patterns for commuters and non-commuters and understand the reasons underlying the differences between these two samples found in this paper. Future research efforts include the development of models for identification of additional causal relationships, tests of applicability across a wide range of contexts, and methodological 
enhancements where time of day choice is treated as a continuous decision variable using hazard-based survival model formulations.

\section{ACKNOWLEDGMENTS}

The authors are grateful to two former graduate research assistants at the University of South Florida, Govardhan Muthyalagari and Abhishankar Parashar, who provided assistance with data preparation and model specification. The authors thank the Florida Department of Transportation District 7 office in Tampa for providing the data set used in this study. This research was partially funded by the Florida Department of Transportation. Comments provided by the anonymous reviewers are gratefully acknowledged. The authors are solely responsible for all errors and omissions. Individuals interested in the data set and/or programs used in this paper should contact the authors.

\section{REFERENCES}

Abkowitz, M.D. (1981) An analysis of the commuter departure time decision. Transportation 10 283-297.

Axhausen, K. and Garling, T. (1992) Activity-based approaches to travel analysis: conceptual frameworks, models, and research problems. Transport Reviews 12 493-517.

Barnard, P.O. and Hensher, D.A. (1992) Joint estimation of a polychotomous discretecontinuous choice system: an analysis of the spatial distribution of retail expenditures. Journal of Transport Economics and Policy XXVI(3) 299-312.

Ben-Akiva, M. and Lerman, S.R. (1985) Discrete Choice Analysis: Theory and Application to Travel Demand, The MIT Press, Cambridge. 
Bhat, C.R. (1996) A hazard-based duration model of shopping activity with nonparametric baseline specification and nonparametric control for unobserved heterogeneity. Transportation Research B 30(1) 189-207.

Bhat, C.R. (1998) A model of post-home arrival activity participation behavior. Transportation Research B 32(6) 387-400.

Bhat, C.R. and Koppelman, F.S. (1999) A retrospective and prospective survey of time-use research. Transportation 26(2) 119-139.

Bhat, C.R. and Misra, R. (1999) Discretionary activity time allocation of individuals between inhome and out-of-home and between weekdays and weekends. Transportation 26(2) 193-229.

Bhat, C.R. and Singh, S.K. (2000) A comprehensive daily activity-travel generation model system for workers. Transportation Research A 34(1) 1-22.

Comte, F. (1998) Discrete and continuous time co-integration. Journal of Econometrics 88(2) 207-226.

Doherty, S.T. and Miller, E.J. (2000) A computerized household activity scheduling survey. Transportation 27(1) 75-97.

Feller, W. (1971) An Introduction to Probability Theory and its Applications, Wiley, New York. Goulias, K.G. (1997) Activity-based travel forecasting: what are some issues? In Texas Transportation Institute (ed) Activity-Based Travel Forecasting Conference, June 2-5, 1996: Summary, Recommendations, and Compendium of Papers, Travel Model Improvement Program, US Department of Transportation, Washington DC 37-49.

Hamed, M. and Mannering, F.L. (1993) Modeling travelers' postwork activity involvement: toward a new methodology. Transportation Science 27(4) 381-394. 
Hanemann, W. M. (1984) Discrete/continuous models of consumer demand. Econometrica 52 $541-561$.

Harvey, A.S. and Taylor, M.E. (2000) Activity settings and travel behavior: a social contact perspective. Transportation 32(1) 53-73.

Hunt, J. D., and Patterson, D.M. (1996) A stated preference examination of time of travel choice for a recreational trip. Journal of Advanced Transportation 30(3) 17-44.

Kasturirangan, K, Pendyala, R.M. and Koppelman, F.S. (2002) Role of history in modeling daily activity frequency and duration for commuters. Transportation Research Record 1807, Journal of Transportation Research Board, TRB, National Research Council, Washington DC 129-136.

Kitamura, R, Yamamoto, T, Fujii, S. and Sampath, S. (1996) A discrete-continuous analysis of time allocation to two types of discretionary activities which accounts for unobserved heterogeneity. In Lesort, J.B. Transportation and Traffic Theory, Elsevier, Oxford 431-453.

Kitamura, R, Chen, C, Pendyala, R.M. and R. Narayanan (2000) Micro-simulation of activitytravel patterns for travel demand forecasting. Transportation 27(1) 25-51.

Lee, L.F. (1983) Generalized econometric models with selectivity. Econometrica 51(2) 507-512. Levinson, D. and Kumar, A. (1995) Activity, travel, and the allocation of time. Journal of the American Planning Association 61(4) 458-470.

Maddala, G.S. (1983) Limited Dependent and Qualitative Variables in Econometrics, Cambridge University Press, Cambridge.

Mahmassani, H. and Chang, G.L. (1985) Dynamic aspects of departure-time choice behavior in a commuting system: theoretical framework and experimental analysis. Transportation Research Record 1037, TRB, National Research Council, Washington DC 88-101. 
Mahmassani, H. and Stephan, D. (1988) Experimental investigation of route and departure time dynamics of urban commuters. Transportation Research Record 1203, TRB, National Research Council, Washington DC 69-84.

Mannering, F, Murakami, E. and Kim, S.G. (1994) Models of travelers' activity choice and home-stay duration: analysis of functional form and temporal stability. Transportation 21(4) 371-392.

Mannering, F.L. and Hensher, D.A. (1987) Discrete-continuous econometric models and their application to transport analysis. Transport Reviews 7(3) 227-244.

Pas, E.I. and Harvey, A.S. (1997) Time use research and travel demand analysis and modeling. In Stopher, P.R. and Lee-Gosselin, M Understanding Travel Behavior in an Era of Change, Elsevier, Oxford 315-338.

Pendyala, R.M, Kitamura, R, Chen, C. and Pas, E.I. (1997) An activity-based micro-simulation analysis of transportation control measures. Transport Policy 4(3) 183-192.

Pendyala, R.M, Kitamura, R. and Reddy, D.V.G.P. (1998) Application of an activity-based travel demand model incorporating a rule-based algorithm. Environment and Planning B: Planning and Design 25 753-772.

Pendyala, R.M, Yamamoto, T. and Kitamura, R. (2002) On the formulation of time space prisms to model constraints on personal activity-travel engagement. Transportation 29(1) 73-94.

Steed, J. and Bhat, C.R. (2000) On modeling the departure time choice for home-based social/recreational and shopping trips. Transportation Research Record 1706, TRB, National Research Council, Washington DC 152-159.

Van Garderen, K.J. (2001) Optimal prediction in log-linear models. Journal of Econometrics 104(1) 119-140. 
Wang, J.J. (1996) Timing utility of daily activities and its impact on travel. Transportation Research A 30 189-206.

Wen, C-H. and Koppelman, F.S. (2000) A conceptual and methodological framework for the generation of activity travel patterns. Transportation Research B 32(1) 5-23.

Yamamoto, T. and Kitamura, R. (1999) An analysis of time allocation to in-home and out-ofhome discretionary activities across working days and non-working days. Transportation 26(2) 211-230. 
Table 1. Tampa Bay 1996 Travel Survey Sample Characteristics

\begin{tabular}{|c|c|c|c|}
\hline & \multirow{2}{*}{\multicolumn{2}{|c|}{ Household Attributes }} & \\
\hline & & & \\
\hline & No. of households & 2386 & \\
\hline & Average household size & 2.3 & \\
\hline & 1 person household & $16.3 \%$ & \\
\hline & 2 person household & $56.5 \%$ & \\
\hline & 3 person household & $14.2 \%$ & \\
\hline & $4+$ person household & $13.0 \%$ & \\
\hline & Average vehicle ownership & 1.8 & \\
\hline & 0 car household & $0.8 \%$ & \\
\hline & 1 car household & $39.7 \%$ & \\
\hline & 2 car household & $42.5 \%$ & \\
\hline & $3+$ car household & $17.0 \%$ & \\
\hline & Dwelling unit type & & \\
\hline & Single-family dwelling unit & $76.2 \%$ & \\
\hline & Multi-family dwelling unit & $10.8 \%$ & \\
\hline & Mobile home & $12.1 \%$ & \\
\hline & Annual Income & & \\
\hline & Low $(<\$ 25 \mathrm{~K})$ & $32.8 \%$ & \\
\hline & Medium $(\$ 25 \mathrm{~K}-\$ 50 \mathrm{~K})$ & $42.3 \%$ & \\
\hline & High $(>\$ 50 \mathrm{~K})$ & $24.9 \%$ & \\
\hline & Person Attributes & Commuter & Non-commuter \\
\hline No. of persons & & 1023 & 1881 \\
\hline Age (in years) & & & \\
\hline Young (16-21) & & $3.8 \%$ & $0.7 \%$ \\
\hline Middle (22-49) & & $64.9 \%$ & $15.0 \%$ \\
\hline Old (50-64) & & $25.4 \%$ & $23.6 \%$ \\
\hline Retired $(\geq 65)$ & & $5.9 \%$ & $60.7 \%$ \\
\hline Employment Statu & & & \\
\hline Full time & & $80.4 \%$ & $7.7 \%$ \\
\hline Part time & & $12.2 \%$ & $4.0 \%$ \\
\hline Average daily mai & enance activity frequency (episodes) & 1.32 & 1.54 \\
\hline Average daily mai & enance activity duration (min) & 92.1 & 224.3 \\
\hline Average duration & a maintenance activity episode (min) & 69.8 & 145.6 \\
\hline
\end{tabular}


Table 2. Non-Commuter Model: Time of Day Affects Activity Duration

\begin{tabular}{|c|c|c|}
\hline Variable & Parameter Estimate & t-statistic \\
\hline \multicolumn{3}{|c|}{ Time of Day Model (Off-peak $=0$ ) } \\
\hline \multicolumn{3}{|c|}{ AM Peak Activity Participation } \\
\hline Constant & 0.3852 & 3.355 \\
\hline Person age $50-64$ years & -0.7530 & -3.418 \\
\hline Full-time employed & 0.6157 & 3.061 \\
\hline Multi-family dwelling unit & -0.4946 & -1.997 \\
\hline \multicolumn{3}{|l|}{ Midday Activity Participation } \\
\hline Constant & 2.4973 & 26.673 \\
\hline Person age $22-49$ years & -0.3409 & -2.951 \\
\hline Person age $50-64$ years & -0.4837 & -3.079 \\
\hline Homemaker & 0.2491 & 1.950 \\
\hline \multicolumn{3}{|l|}{ PM Peak Activity Participation } \\
\hline Constant & 1.2516 & 3.811 \\
\hline Person age $50-64$ years & -0.3428 & -1.815 \\
\hline Household size $=1$ & 0.2019 & $1.415^{\mathrm{a}}$ \\
\hline Licensed driver & -0.4600 & $-1.433^{\mathrm{a}}$ \\
\hline \multicolumn{3}{|c|}{ Log-Linear Duration Model } \\
\hline Constant & 1.8907 & 25.633 \\
\hline Household size $=1$ & 0.0785 & 2.103 \\
\hline Household with no child & 0.0796 & $1.515^{\mathrm{a}}$ \\
\hline Homemaker & -0.0976 & -2.125 \\
\hline Person age $22-49$ years & -0.1331 & -2.878 \\
\hline Annual household income $<\$ 25,000$ & 0.1387 & 5.106 \\
\hline $\begin{array}{l}\text { History of maintenance activity engagement } \\
\text { up to the current activity (min) }\end{array}$ & 0.3431 & 2.665 \\
\hline Time of day: AM Peak (7:15-9:15 AM) & -0.2719 & -3.882 \\
\hline Time of day: Midday (9:16 AM-3:15 PM) & -0.5718 & -9.052 \\
\hline Time of day: PM Peak (3:16-6:15 PM) & -0.4003 & -6.214 \\
\hline \multicolumn{3}{|c|}{ Log-Linear Model Error Term } \\
\hline Standard deviation, $\sigma$ & 0.7385 & 43.744 \\
\hline \multicolumn{3}{|c|}{ Error Correlation Estimates } \\
\hline$\rho_{\text {midday-duration }}$ & -0.5984 & -16.895 \\
\hline
\end{tabular}


Table 3. Non-Commuter Model: Activity Duration Affects Time of Day

\begin{tabular}{|c|c|c|}
\hline Variable & Parameter Estimate & t-statistic \\
\hline \multicolumn{3}{|c|}{ Time of Day Model (Off-peak =0) } \\
\hline \multicolumn{3}{|c|}{ AM Peak Activity Participation } \\
\hline Constant & -1.3767 & -5.186 \\
\hline Activity Duration & 1.8034 & 16.558 \\
\hline Person age $22-49$ years & -0.5770 & -2.127 \\
\hline Person age 50-64 years & -0.8484 & -3.794 \\
\hline Full-time employed & 0.3760 & $1.573^{\mathrm{a}}$ \\
\hline Multi-vehicle household (>1 vehicle) & 0.3138 & 2.080 \\
\hline Multi-family dwelling unit & -0.3994 & $-1.513^{\mathrm{a}}$ \\
\hline \multicolumn{3}{|l|}{ Midday Activity Participation } \\
\hline Constant & 3.0425 & 25.048 \\
\hline Person age $22-49$ years & -0.6771 & -3.870 \\
\hline Person age $50-64$ years & -0.4490 & -3.736 \\
\hline \multicolumn{3}{|l|}{ PM Peak Activity Participation } \\
\hline Constant & -0.6244 & -2.735 \\
\hline Activity Duration & 1.6860 & 17.756 \\
\hline Person age $22-49$ years & -0.3770 & -1.688 \\
\hline Person age $50-64$ years & -0.3843 & -2.136 \\
\hline \multicolumn{3}{|c|}{ Log-Linear Duration Model } \\
\hline Constant & 1.3435 & 56.541 \\
\hline Household size $=1$ & 0.0583 & 1.818 \\
\hline Household with no child & 0.4021 & 8.290 \\
\hline Homemaker & -0.0687 & -1.838 \\
\hline Person age $22-49$ years & -0.1467 & -3.259 \\
\hline Annual household income $<\$ 25,000$ & 0.0809 & 3.401 \\
\hline $\begin{array}{l}\text { History of maintenance activity engagement } \\
\text { up to the current activity (min) }\end{array}$ & 0.1840 & 1.853 \\
\hline \multicolumn{3}{|c|}{ Log-Linear Model Error Term } \\
\hline Standard deviation, $\sigma$ & 0.8296 & 48.542 \\
\hline \multicolumn{3}{|c|}{ Error Correlation Estimates } \\
\hline$\rho_{\text {midday-duration }}$ & -0.8938 & -52.111 \\
\hline
\end{tabular}


Table 4. Commuter Model: Time of Day Affects Activity Duration

\begin{tabular}{|c|c|c|}
\hline Variable & Parameter Estimate & t-statistic \\
\hline \multicolumn{3}{|c|}{ Time of Day Model (Off-peak =0) } \\
\hline \multicolumn{3}{|c|}{ AM Peak Activity Participation } \\
\hline Constant & -0.2598 & -0.911 \\
\hline Full-time employed & -0.7408 & -2.647 \\
\hline Household with no child & -0.6896 & -3.071 \\
\hline \multicolumn{3}{|l|}{ Midday Activity Participation } \\
\hline Constant & 0.9905 & 3.912 \\
\hline Household size $>3$ & -0.3516 & -2.390 \\
\hline Person age $22-49$ years & -0.9610 & -3.689 \\
\hline Person age $50-64$ years & -0.9828 & -3.474 \\
\hline Part-time employed person & 0.9656 & 4.097 \\
\hline Homemaker & 2.4549 & 2.341 \\
\hline \multicolumn{3}{|l|}{ PM Peak Activity Participation } \\
\hline Constant & 0.8773 & 3.455 \\
\hline Person age $22-49$ years & -0.5342 & -2.027 \\
\hline Person age $50-64$ years & -0.5999 & -2.122 \\
\hline Part-time employed person & 0.5153 & 2.167 \\
\hline $\begin{array}{l}50-64 \text { year old non-worker in a multi-adult } \\
\text { household }\end{array}$ & -1.3329 & -1.721 \\
\hline \multicolumn{3}{|c|}{ Log-Linear Duration Model } \\
\hline Constant & 1.5781 & 31.573 \\
\hline$\$ 25,000 \leq$ Annual hhld income $\leq \$ 50,000$ & -0.0660 & -1.862 \\
\hline Full-time employed & -0.1186 & -2.715 \\
\hline Person age $22-49$ years & -0.0984 & -2.497 \\
\hline Household size $=1$ & 0.1211 & 1.993 \\
\hline Household size $>3$ & -0.0991 & -2.503 \\
\hline Time of Day: AM Peak (7:15-9:15 AM) & -0.3177 & -4.614 \\
\hline Time of Day: Off Peak (6:16 PM-7:14 AM) & 0.1040 & 2.585 \\
\hline \multicolumn{3}{|c|}{ Log-Linear Model Error Term } \\
\hline Standard deviation, $\sigma$ & 0.6378 & 53.724 \\
\hline \multicolumn{3}{|c|}{ Error Correlation Estimates } \\
\hline All correlation estimates statistically insignif & ant at $\alpha=0.05$ level. & \\
\hline
\end{tabular}


Table 5. Commuter Model: Activity Duration Affects Time of Day

\begin{tabular}{|c|c|c|}
\hline Variable & Parameter Estimate & t-statistic \\
\hline \multicolumn{3}{|c|}{ Time of Day Model (Off-peak =0) } \\
\hline \multicolumn{3}{|c|}{ AM Peak Activity Participation } \\
\hline Constant & 0.3102 & 0.487 \\
\hline Activity Duration & -0.9890 & -5.548 \\
\hline Household size $=2$ & -0.5040 & -1.824 \\
\hline Worker (full or part time) & -1.6301 & -3.733 \\
\hline Person age $22-49$ years & 1.3544 & 2.372 \\
\hline Person age $50-64$ years & 1.3541 & 2.316 \\
\hline Part-time employed person & 1.0982 & 3.084 \\
\hline \multicolumn{3}{|l|}{ Midday Activity Participation } \\
\hline Constant & 1.4140 & 4.476 \\
\hline Activity Duration & -0.1753 & $-1.483^{\mathrm{a}}$ \\
\hline Homemaker & 1.9565 & 1.754 \\
\hline Worker & -1.2594 & -4.689 \\
\hline Part-time employed person & 1.1688 & 5.041 \\
\hline \multicolumn{3}{|l|}{ PM Peak Activity Participation } \\
\hline Constant & 0.9420 & 3.155 \\
\hline Activity Duration & -0.2934 & -2.728 \\
\hline Multi-vehicle household ( $>1$ vehicle) & 0.3789 & 2.178 \\
\hline Annual household income $<\$ 25,000$ & 0.3116 & 1.817 \\
\hline Full-time employed person & -0.5763 & -2.737 \\
\hline \multicolumn{3}{|c|}{ Log-Linear Duration Model } \\
\hline Constant & 1.5677 & 32.165 \\
\hline$\$ 25,000 \leq$ Annual hhld income $\leq \$ 50,000$ & -0.0599 & -1.669 \\
\hline Full-time employed person & -0.0957 & -2.207 \\
\hline Person age $22-49$ years & -0.1084 & -2.677 \\
\hline Household size $=1$ & 0.1197 & 1.894 \\
\hline Household size $>3$ & -0.1040 & -2.587 \\
\hline \multicolumn{3}{|c|}{ Log-Linear Model Error Term } \\
\hline Standard deviation, $\sigma$ & 0.6457 & 52.539 \\
\hline \multicolumn{3}{|c|}{ Error Correlation Estimates } \\
\hline All correlation estimates statistically insigr & ant at $\alpha=0.05$ level. & \\
\hline
\end{tabular}


Table 6. Measures of Fit for Joint Timing-Duration Models

\begin{tabular}{lcccc}
\hline & \multicolumn{2}{c}{ Non-Commuter Model } & \multicolumn{2}{c}{ Commuter Model } \\
\cline { 2 - 5 } Summary Statistic & $\begin{array}{c}\text { Time of Day } \\
\text { Affects } \\
\text { Duration }\end{array}$ & $\begin{array}{c}\text { Duration } \\
\text { Affects Time } \\
\text { of Day }\end{array}$ & $\begin{array}{c}\text { Time of Day } \\
\text { Affects } \\
\text { Duration }\end{array}$ & $\begin{array}{c}\text { Duration } \\
\text { Affects Time } \\
\text { of Day }\end{array}$ \\
\hline $\begin{array}{c}\text { Log-likelihood at zero, } \mathrm{L}(0)^{\mathrm{a}} \\
\text { Log-likelihood at sample } \\
\text { shares, } \mathrm{L}(\mathrm{C})^{\mathrm{b}}\end{array}$ & -7072.00 & -7072.00 & -3216.60 & -3216.60 \\
$\begin{array}{c}\text { Log-likelihood at convergence, } \\
\text { L }(\beta)\end{array}$ & -5756.11 & -5756.11 & -3058.38 & -3058.38 \\
$\begin{array}{c}\text { Number of parameters, } \mathrm{k} \\
\text { Number of observations, } \mathrm{N}\end{array}$ & -5584.14 & -5316.82 & -2990.86 & -2990.60 \\
$\begin{array}{c}\text { Adjusted likelihood ratio index } \\
\text { at zero, } \bar{\rho}_{0}^{2}\end{array}$ & 22 & 21 & 21 & 22 \\
$\begin{array}{c}\text { Adjusted likelihood ratio index } \\
\text { at sample shares, } \bar{\rho}_{\mathrm{c}}^{2}\end{array}$ & 0.207 & 2899 & 1351 & 1351 \\
\hline
\end{tabular}

${ }^{\mathrm{a}}$ The log-likelihood at zero corresponds to the likelihood function value of the joint model with no variables in the MNL time of day model, and with only the constant and variance (standard deviation) term in the log-linear duration equation. All correlation terms are zero.

${ }^{b}$ The log-likelihood at sample shares corresponds to the likelihood function value of the joint model with only alternative specific constants in the MNL time of day model, and with only the constant and variance term in the log-linear duration equation. All correlation terms are zero.

${ }^{\mathrm{c}}$ The number of parameters $(\mathrm{k})$ does not include the constant and variance term in the log-linear duration model. 


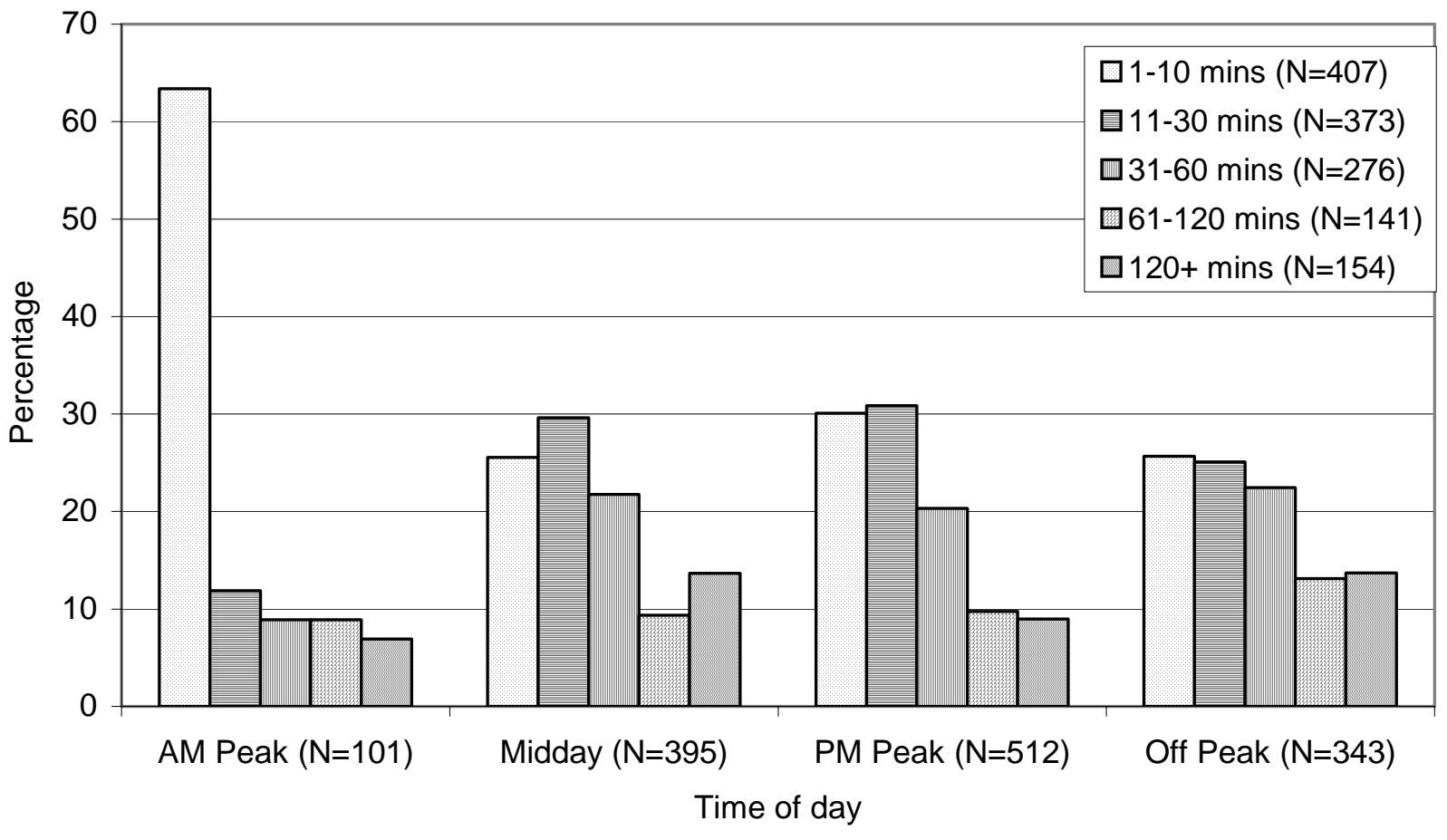

Figure 1. Maintenance Activity Duration Distribution by Time of Day (Commuters) 


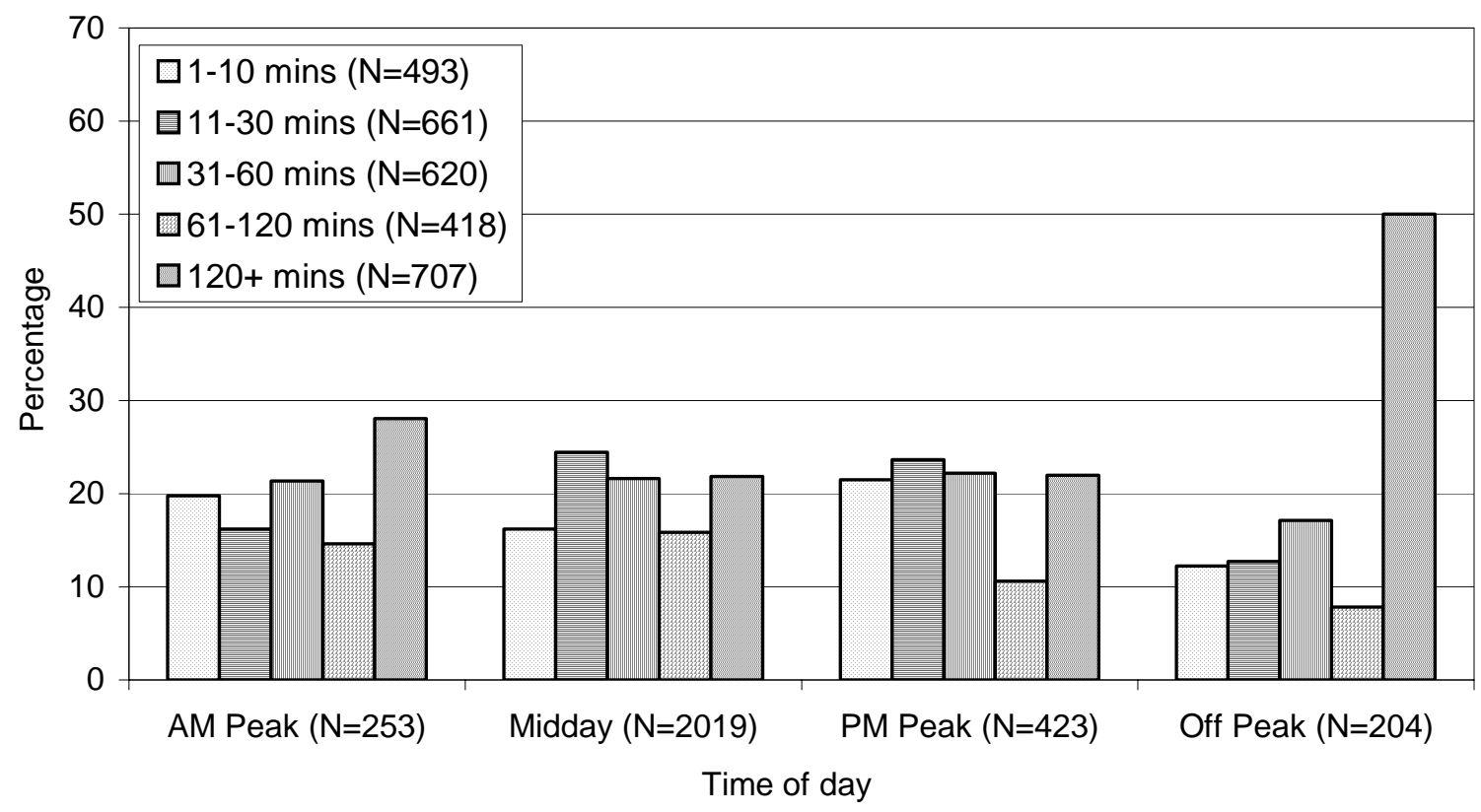

Figure 2. Maintenance Activity Duration Distribution by Time of Day (Non-commuters) 


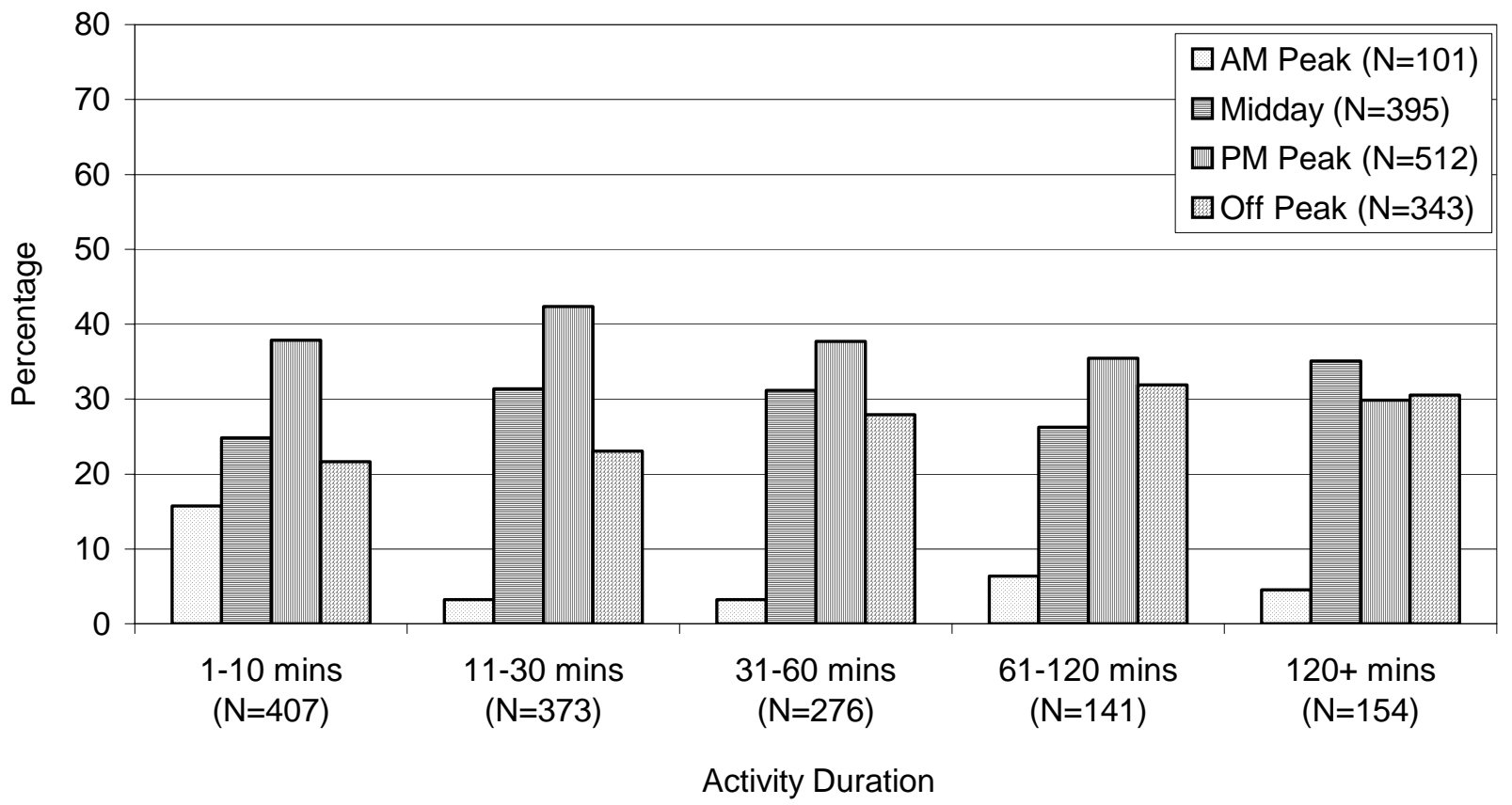

Figure 3. Time of Day Distribution by Activity Episode Duration (Commuters) 


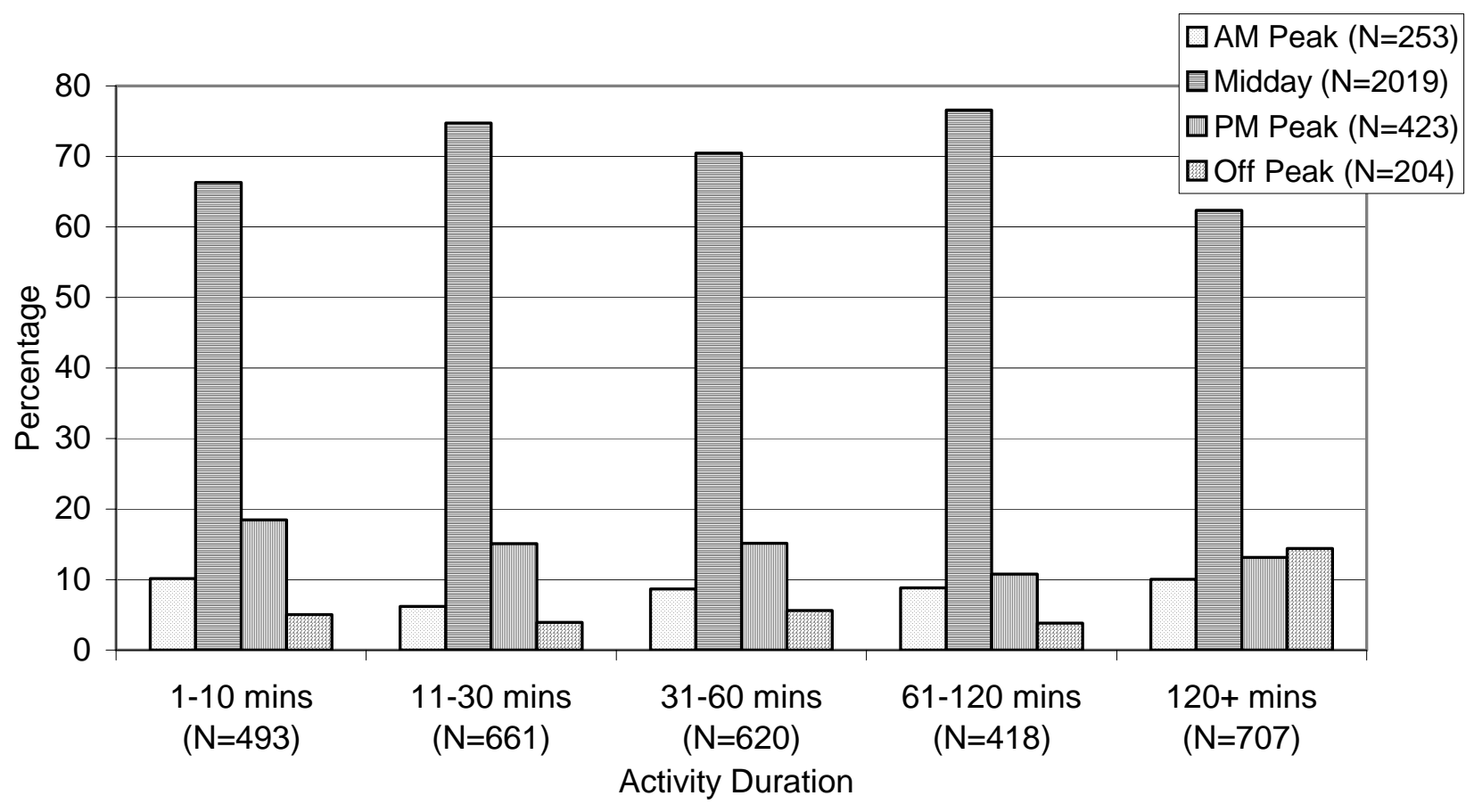

Figure 4. Time of Day Distribution by Activity Episode Duration (Non-commuters) 